\title{
African Traditional Medicine Based Immune Boosters and Infectious Diseases: A Short Commentary
}

\section{Mlungisi Ngcobo' and Nceba Gqaleni}

${ }^{1}$ Traditional Medicine Laboratory School of Nursing and Public Health, College of Health Sciences, University of KwaZulu Natal, South Africa ${ }^{2}$ Department of Public Management and Economics, Faculty of Management Sciences, Durban University of Technology, South Africa

\begin{abstract}
Introduction: Developed from centuries of oral knowledge passed from generation to generation, African traditional medicines (ATM) are at a crossroads. In order for these ATMs to evolve with time similarly to modern medical sciences, there is an urgent need to scientifically evaluate their mechanism of action, safety and efficacy.

Case report: In this short commentary we report on the progress we have made in scientifically evaluating traditional herbal immune boosters at a period where the African continent finds itself ravaged by a plethora of infectious diseases.

Discussion: While the South African government has gone a long way in building an effective modern health system, many people still use ATMs such as immune boosters for a variety of infectious diseases and general wellbeing. The high prevalence of HIV infections is one of the reasons that have led to an increase in the use of ATMs which purport to have immune boosting capabilities. In our laboratory we have developed various models and carried out extensive in vitro and in vivo biochemical and molecular experiments on the immunomodulatory effects of these immune boosters and have shown that these products can modulate the expression and secretion of both cytokines and chemokines. Therefore the potential uses of immune boosters in clinical medicine does not only apply in treating immunodeficiency caused by HIV but can be useful in managing tuberculosis (TB), various forms of cancer and various other diseases that can be managed by modulating the immune response. Such research studies are very promising and present opportunities for further clinical studies to develop unique ATMs.
\end{abstract}

Conclusion: As medicines meant to stimulate the body's immune system to defend itself, immune boosters can be useful alone or as immune adjuvants along with pharmaceutical drugs. Therefore further studies on the effectiveness of these immune boosters are justified along with those that will ensure that there are no drug interactions with other pharmaceutical products.

Keywords: African traditional medicines; Immune boosters; Infectious diseases

\section{Introduction}

Created by complicated social, political and economic processes, medical provision in Africa has come to take many forms. Africans developed their own health and healing traditions, including ways of recognizing, classifying and treating disease and illness [1]. Within the different factors thought to cause sickness, biological factors are regarded as the malfunctioning of the organism, a natural process or even an inherited disorder. This is not only a recognition of the molecular genetic bases of diseases as explained in modern biomedical sciences but also that the African healing system has always developed means to mitigated such sicknesses. Most importantly, the philosophy of African traditional healing centers around restoring the body's defence systems, strengthening both mind and body through combinations of physical and mental interventions. While medical scientists may regard this approach as unscientific, users of TM appreciate that its rationality in the cultural context it operates [2].

From this concept of healing the body, the most important forms of African traditional medicines (ATMs) have been developed. These ATMs are commonly known as immune boosters, immunomodulators or energy tonics. These energy medicines are used to stimulate the body's immune and hormonal systems and provide effective strategies to reduce stress; thus reducing the assault on the immune system caused by the production of stress-related bio-chemicals [3]. An immune booster is described as an additional immunizing agent given to increase and sustain the immune response of the body. Others define immune boosters as products which can either target the body's innate defensive response or the invading microorganisms [4]. Interestingly the concept of boosting the immune system is now part of modern medicine. Obviously these modern immune boosters are very different in their form when compared to ATMs immune boosters which are formulated using different crude extracts of medicinal plants. The main concern about the ATMs immune boosters and related products is that the ATMs sector remains largely unregulated and their use, safety and efficacy remain undocumented. It is in the context of the known burden of infectious diseases in Africa that our research on all forms of ATMs including immune boosters become a matter of public health. This is in addition to the fact that the number of traditional health practitioners (THPs) is still substantially higher than those of medical doctors in the majority of Sub-Saharan Africa with the ratio of traditional healers to the population at approximately 1:500, in contrast to the doctor to population ratio of 1:40 $000[5,6]$. Table 1 below shows a few examples of ratios of THPs and doctors in various countries of Africa.

*Corresponding author: Nceba Gqaleni, Department of Public Management and Economics, Faculty of Management Sciences, Durban University of Technology, South Africa, Tel: +27 31373 2000; E-mail: nceba5850@gmail.com

Received October 08, 2015; Accepted November 24, 2015; Published November 28,2015

Citation: Ngcobo M, Gqaleni N (2015) African Traditional Medicine Based Immune Boosters and Infectious Diseases: A Short Commentary. J Mol Biomark Diagn 7 265. doi:10.4172/2155-9929.1000265

Copyright: $\odot 2015 \mathrm{Ngcobo} \mathrm{M}$, et al. This is an open-access article distributed under the terms of the Creative Commons Attribution License, which permits unrestricted use, distribution, and reproduction in any medium, provided the original author and source are credited. 


\begin{tabular}{|l|c|c|}
\hline African countries & Ratio of THPs to population & $\begin{array}{c}\text { Ratio of Medical Doctors to } \\
\text { population }\end{array}$ \\
\hline Zimbabwe & $1: 600$ & $1: 6250$ \\
\hline Swaziland & $1: 100$ & $1: 10000$ \\
\hline Ghana & $1: 200$ & $1: 20000$ \\
\hline Uganda & $1: 700$ & $1: 25000$ \\
\hline Tanzania & $1: 400$ & $1: 33000$ \\
\hline Mozambique & $1: 200$ & $1: 50000$ \\
\hline South Africa* & $5: 1000$ & $50: 100000$ \\
\hline
\end{tabular}

Sources: Abdool-Karim et al. [5], George et al., [6]

Table 1: Sample ratio of THPs compared with the ratio of medical doctors to the population.

\section{Use of immune boosters against infectious diseases}

Most of the studied herbal medicinal extracts are made of a single plant while most of the ATMs products are made up of two or more plants mixed together. Herbalists have known for centuries the value of using a combination of herbal remedies, single extracts and combined extracts to switch on the body's defence mechanisms and self-healing and protective processes [7]. But research on ATMs is embroiled in all types of politics and contestations about indigenous knowledge and science in relation to medicinal plants. Such contestations surround the testing of ATMs as plant medicines, rather than as purely pharmacological components. A study of traditional medicines was seen as unscientific and predicated, based on the beliefs of people rather than rational evidence [8]. The reality is that this research on multi-herbal immune boosters is based on the need to find empirical evidence of their safety and efficacy. These complex mixtures of compounds may have activity on different targets and may even have synergistic effects in the in vivo system. Therefore immune boosters are inherently nonspecific in nature but can act both through the innate and specific arms of immune response. These immune boosters can be used both by healthy individuals and persons with impaired immune systems. In healthy individuals they are expected to act as prophylactic agents and in individuals with impairment of the immune response as immunotherapeutic agents [4].

The potential uses of immune boosters in clinical medicine does not only apply in treating immunodeficiency caused by HIV and AIDS but can be useful in managing tuberculosis (TB), various forms of cancer and various other diseases that can be managed by modulating the immune response. This was demonstrated by controlled observation clinical studies using Phela, a traditional South Africa immune booster formulated by traditional healers, conducted on $500 \mathrm{HIV}$ positive and AIDS patients. The results showed an increase in the patients' appetites, $23 \%$ increase in weight gains, $80 \%$ decrease in viral loads and $200 \%$ increase in $\mathrm{CD}^{4+}$ cell counts. The overall quality of life of the patients increased, some from as low as $30 \%$ to $100 \%$. These results were indicative of the immune boosting properties of Phela [9]. Regarding the immunomodulatory effects of immune boosters, these ATMs alter the activities of the immune system via the dynamic regulation of informational molecules - cytokines, hormones, neurotransmitters, and other peptides [10]. We have also carried out extensive in vitro research experiments on the immunomodulatory effects of ATMs immune boosters and have shown that these products can modulator both inflammatory and anti-inflammatory cytokines and also chemokines. The in vitro studies on the effects of ATMs immune booster were under taken and these looked at the immune toxicology, genotoxicology, free radical scavenging, lipid peroxidation, secretion of inflammatory cytokines, secretion of chemokines, chemotaxis, levels of soluble interleukin 2 receptors, and transcriptional activity of NF- $\kappa \beta$ in PBMCs from normal human blood and THP-1 monocytes. Models used included cyclosporine immunocompromised, peptidoglycan from Staphylococcus aureus and phytohaemagglutinin pretreated PBMCs. For the THP-1 monocytes assays, normal and lipopolysaccharide (LPS) from Salmonella typhosa (S. typhosa) stimulated monocytes were used. Some of the research results from these studies have been published in various peer review journals $[11,12]$ and further publications have been submitted to other peer reviewed scientific journals for future publication. Interestingly, our research has also shown that minimal doses of these immune boosters are required to modulate cytokines and chemokines secretion. This disputes the assertion by Gertsch et al., [13] that in vitro bioassays on immunomodulators use concentrations that are physiologically unreasonable.

Research on the in vitro anti-HIV activities of some of these immune boosters has been undertaken largely because of unproven claims about such activities [11]. To study these effects, the protective effects against HIV killing of infected MT- 4 cells were tested using the XTT assay and antiviral activity was measured using an HIV-1 viral load assay. Even though some of these immune boosters demonstrated promising protective effects for T lymphocytes against HIV infection and reduced viral loads comparative to azidothymidine (AZT), these effects did not supersede the their potential as immunomodulators. In vivo experiments using bacteria infected rat models as a starting point are also ongoing in our research laboratory to further develop the scientific knowledge and mechanisms of action of these ATMs immune boosters. Our abilities to conduct world class clinical trials on standardized traditional medicines have been demonstrated by the recently published results of the Sutherlandia frutescens phase 1 and 2 studies [14]. Further clinical trials of some of multi-herbal immune boosters are already in the planning phase and applications for funding have been submitted. Our research endeavors are supported by results obtained by peers in other institutions doing research on traditional medicines. Such studies demonstrate the potential benefits of ATMs immune boosters and moving forward, we aim to conduct our own gold standard clinical trials of ATMs products under development. South Africa has for a long time demonstrated the ability to conduct such high caliber clinical trials and we aim to apply those available skills in studies of ATMs.

The applicability of ATMs immune boosters is not only limited to HIV and AIDS but we have demonstrated, through various ongoing research studies, that they can be effective in modulating the immune response to TB and cancer cells. Some of the immune boosters we are researching have shown the ability modulate interferon gamma $(\mathrm{IFN} \gamma)$ and tumour necrosis factor alpha (TNFa) secretion in mock mycobacterium TB infected THP-1 monocytes thus providing interesting leads in towards the discovery of medicines that can be used with current anti-TB drugs. Other ATMs immune boosters have not only shown selective cytotoxicity to cancerous cells than primary human normal blood cell lines, their ability to modulate the secretion of inflammatory cytokines and chemokines can be useful in enhancing the immune response against cancer cells. Another ATMs product used to treat Kaposi's sarcoma (KS), an AIDS defining cancer caused by Kaposi's sarcoma-associated herpes virus (KSHV), significantly reactivated latent $\mathrm{KSHV}$ in primary effusion lymphoma (PEL) cell lines thus potentially alerting immune cells to the presence of infection. Such research studies are very promising and present opportunities for medical practitioners to prescribe these ATMs along with pharmaceutical drugs for more effective treatment of infectious diseases. Obviously there is a need for caution when prescribing pharmaceuticals with herbal medicine because there is a likelihood for 
drug interactions. The dangers of drug interactions were demonstrated by studies that showed that Sutherlandia frutescens had potential inhibitory effects on various cytochrome P450 enzymes [15,16].

\section{Models for studying immune boosters}

Many advances have been made recently in the understanding of the functioning of the immune system. Whether immunomodulators enhance or suppress immune responses can depend on a number of factors, including dose, route of administration, and timing of administration. This response can also depend on their mechanism of action or site of activity [4]. Therefore in choosing research models to study immunomodulators, a careful and precise process must be followed in order to ensure that the results of such research reflect the absolute nature and mechanisms of action of the compounds in question. Knowledge of the specific components of cytokine networks and signaling pathways and their role in regulation of immune responses is also important in designing strategies to augment these responses [17]. In our research endeavors we have tried, working hand in hand with indigenous knowledge holders and traditional healers, to create the most appropriate models for studying ATMs immune boosters. Whole blood and peripheral blood mononuclear cells from samples obtained and certified by the South African Blood National Services (SANBS) as clear of any infection are utilized as detailed in the previous paragraphs. Other in vitro models include PEL cell lines latently infected with KSHV (BCBL-1 and BC-3) and a virus-negative Burkitt's lymphoma cell line (BJAB). We have also collaborated with international institutions to study our ATMs using latently infected KSHV mice models. Standard in vitro and animals models of TB are utilized to study ATMs specially prepared microbiology studies. However, the ultimate goal is to have enough resources to conduct clinical studies of all immune boosters with defined formulations that have demonstrated enough potential in in vitro and animal model studies.

\section{Biomarkers for African traditional medicines}

In the development of pharmacological drugs, plasma biomarkers are used in biokinetic studies to trace the metabolism of each dose after ingestion. Biomarkers are useful in the determining proof of ingestion and bioavailability of the product, change in dose, potential host problems, therapeutic end points, toxicity and in evaluating for variations in response among individuals [18]. While standard methods to identify biomarkers for drugs with single active entities are readily available, identification of biomarkers for ATMs is complicated by the fact the majority of these products are herbal mixtures with multitudes of active compounds. Even though this is a challenge, it is important that these biomarkers are identified for each ATM product in order to full understand their efficacy and safety. Using chromatographic methods, peak area per unit volume to derive pharmacokinetics of unknown compounds can be used as demonstrated in the development of Phela [18]. This is one of many examples that can be used to identify biomarkers for ATMs and more simplified methods need to be developed.

\section{Conclusion}

Medicinal products from ATMs meant to arm the body's own defenses form the bases for African medicinal healing systems. Even though these so called immune boosters have been used for centuries, their current use in era of high rates of infectious diseases has raised questions about their effectiveness. While the influence of modern biomedical sciences and its germ based theory of infectious diseases has crossed over to traditional healing, it is important to avoid the temptation of claiming the effectiveness of ATMs such as immune boosters based on their antimicrobial activity. As medicines meant to stimulate the body's immune system to defend itself, ATMs immune boosters can be useful as immune adjuvants along with pharmaceutical drugs. In order to prove the effectiveness of such combination therapies, suitable research models must be used to fast-track studies on immune boosters along with ensuring that there are no drug interactions.

\section{References}

1. Mokaila A (2001) Traditional vs. Western Medicine-African Context. Drury University, pringfield, Missouri.

2. Okpako DT (1999) Traditional African medicine: theory and pharmacologyviewpoint. TiPS 20: 482-485.

3. Cocks M, Moller $V$ (2002) Use of indigenous and indigenized medicines to enhance personal well-being: a South African case study. Soc Sci Med 54 387-397.

4. Agarwal SS, Singh VK (1999) Immunomodulators: a review of studies on Indian medicinal plants and synthetic plants. PINSA B65 3 \& 4: 179-204.

5. Abdool Karim SS, Ziqubu-Page TT, Arendse R (1994) Bridging the Gap: Potential for a health care partnership between African traditional healers and biomedical personnel in South Africa (supplement). S Afr Med J 84: 1-16.

6. George G, Chitindingu E, Gow J (2013) Evaluating traditional healers knowledge and practices related to HIV testing and treatment in South Africa. BMC Int Health Hum Rights 13: 1-7.

7. Busia K (2005) Medical provision in Africa- past and present. Phytother Res 19: $919-923$

8. Gibson D (2011) Ambiguities in the making of an African Medicine: clinical trials of Sutherlandia frutescens (L.) R. $\mathrm{Br}^{1}$ (Lessertia frutescens). Afr Soc Rev 15 124-137.

9. Matsabisa MG (2006) The preclinical and clinical evaluation of a traditional medicinal product, Phela, a potential immune modulator: A case study of scientific validation of African Traditional Medicines. Proceedings of $12^{\text {th }}$ International Congress of Ethnopharmacology, India.

10. Spelman K, Burns JJ, Nichols D, Winters N, Ottersberg S, et al. (2006) Modulation of cytokine expression by traditional medicines: a review of herbal immunomodulators. Altern Med Rev 11: 128- 150.

11. Gqaleni N, Ngcobo M, Parboosing R, Naidoo A (2012) In vitro testing of African traditional medicines for cytotoxic, immune modulatory and anti-HIV activities. Afr J Tradit Complement Altern Med 9: 2-11.

12. Ngcobo M, Gqaleni N, Ndlovu V, Serumula M, Sibiya N (2016) Immunomodulatory effects of Umakhonya ${ }^{\circledR}$ : A South African commercial traditional immune booster. S Afr J Bot 102: 26-32.

13. Gertsch J, Taylor P, Viveros-Paredes JM (2011) Plant immunostimulantsscientific paradigm or myth? J Ethnopharmacol 136: 385-391.

14. Wilson D, Goggin K, Williams K, Gerkovich MM, Gqaleni N, et al. (2015) Consumption of Sutherlandia frutescens by HIV-Seropositive South African adults: An adaptive double-blind randomized placebo controlled trial. PLOS ONE 10: 1-14

15. Mills E, Cooper C, Seely D, Kanfer I (2005) African herbal medicines in the treatment of HIV: Hypoxis and Sutherlandia. An overview of evidence and pharmacology. Nutr $\mathrm{J} 4: 19$.

16. Prevoo D, Swart P, Swart AC (2008) The influence of Sutherlandia frutescens on adrenal steroidogenic cytochrome P450 enzymes. J Ethnopharmacol 118: 118-126.

17. Tzianabos AO (2000) Polysaccharide immunomodulators as therapeutic agents: Structural aspects and biologic function. Clin Microbiol Rev 13: 523-533.

18. Lekhooa M, Walubo A, Du Plessis JB, Matsabisa MG (2012) Evaluation of traditional medicines II: the use of metabolite peak-kinetics to monitor Phela in rat plasma. Afr J Tradit Complement Altern Med 9: 73-80. 